\title{
ON MEROMORPHIC FUNCTIONS DEFINED BY A NEW CLASS OF LIU-SRIVASTAVA INTEGRAL OPERATOR
}

\section{SYED GHOOS ALI SHAH ${ }^{1}$, SAIMA NOOR ${ }^{2}$, MASLINA DARUS ${ }^{3, \dagger, *}$, WASIM UL HAQ ${ }^{4}$, SAQIB HUSSAIN ${ }^{1}$}

\footnotetext{
${ }^{1}$ Department of Mathematics, COMSATS University Islamabad, Abbottabad Campus 22060, Pakistan

${ }^{2}$ Department of Basic Sciences, Preparatory year deanship, King Faisal University, Hofuf 31982 Al Ahsa, Saudia Arabia

${ }^{3}$ Department of Mathematical Sciences, Universiti Kebangsaan Malaysia, Bangi 43600, Selangor, Malaysia

${ }^{4}$ Department of Mathematics, Abbottabad University of Science and Technology, Abbottabad 22010,
} Pakistan

${ }^{*}$ Corresponding author: maslina@ukm.edu.my

\begin{abstract}
In this work, we introduce and explore certain new subclasses of meromorphic functions. We aim to study some important properties such as coefficient estimates, growth rate and partial sums for these newly defined subclasses. It is important to mentioned that our results are generalization of number of existing results.
\end{abstract}

\section{Introduction}

Let $\sum_{p}$ denote the class of $p$-valent meromorphic function of the form:

$$
\lambda(\omega)=\frac{1}{\omega^{p}}+\sum_{t=p}^{\infty} a_{t} \omega^{t},
$$

Received September $5^{\text {th }}, 2020$; accepted September $30^{\text {th }}, 2020$; published October $28^{\text {th }}, 2020$.

2010 Mathematics Subject Classification. Primary 30C45, Secondary 30C50.

Key words and phrases. integral operator; meromorphic function; starlike function.

${ }^{\dagger}$ This author is supported by the grant number GUP-2019-032.

(C)2020 Authors retain the copyrights of their papers, and all open access articles are distributed under the terms of the Creative Commons Attribution License. 
which are analytic in the punctured open unit disc $U^{*}=\{\omega: \omega \in \mathbb{C}$ and $0<\{\omega\}<1\}=U-\{0\}$, where $U=U^{*} \cup\{0\}$.

Here we are listing some important subclasses of meromorphic functions which will be used in our subsequal work. In 1936, Roberston [23] introduced the classes of meromorphic starlike and meromorphic convex functions of order $\alpha$. By $\sum^{M S}(\alpha)$ we mean the subclass of $\sum_{1}$ consisting of all meromorphic starlike functions of order $\alpha$. Analytically

$$
\lambda(\omega) \in \sum^{M S}(\alpha) \Leftrightarrow \Re\left(\frac{\omega \lambda^{\prime}(\omega)}{\lambda(\omega)}\right)<-\alpha, \quad\left(0 \leq \alpha<1 ; \omega \in U^{*}\right) .
$$

A closely related class of meromorphic convex functions of order $\alpha$ is denoted by $\sum^{M C}(\alpha)$ and defined as:

$$
\lambda(\omega) \in \sum^{M C}(\alpha) \Leftrightarrow-\omega \lambda^{\prime}(\omega) \in \sum^{M S}(\alpha) .
$$

In 1952, Kaplan [16] introduced and studied an important class of analytic functions in the open unit disc $U$ known as close-to-convex functions. A function $\lambda$ belongs to $\sum_{1}$ is in class $\sum^{M K}(\alpha, \beta)$, of meromorphic close-to-convex functions of order $\alpha$ and type $\beta$, if there exist $\delta(\omega) \in \sum^{M S}(\beta)$ and

$$
\Re\left(\frac{\omega \lambda^{\prime}(\omega)}{\delta(\omega)}\right)<-\alpha .
$$

Many differential and integral operators can be written in terms of convolution of certain holomorphic functions. Let $\delta(\omega) \in \sum_{p}$ and having series representation of the form

$$
\delta(\omega)=\frac{1}{\omega^{p}}+\sum_{t=0}^{\infty} b_{t} \omega^{t}
$$

then convolution (Hadamard product) is denoted by $\lambda * \delta$ and defined as:

$$
(\lambda * \delta)(\omega)=\frac{1}{\omega^{p}}+\sum_{t=0}^{\infty} a_{t} b_{t} \omega^{t}=(\delta * \lambda)(\omega),
$$

where $\lambda(\omega)$ as given by (1.1).

Following the current work of Liu and Srivastava [18] (see also [1]- [6]), now we defined the integral operator given below

$$
M_{p}^{m}(a, b) \lambda(\omega)=\frac{1}{\omega^{p}}+\sum_{t=p}^{\infty}\left[\frac{a}{a+b(p+t)}\right]^{m} a_{t} \omega^{t} \quad(a b>0 ; p \in \mathbb{N}) .
$$

The above integral operator converts into the following operator when $p=1$

$$
M_{1}^{m}(a, b) \lambda(\omega)=\frac{1}{\omega}+\sum_{t=p}^{\infty}\left[\frac{a}{a+b(1+t)}\right]^{m} a_{t} \omega^{t} \quad(a>0, b \geq 0, m \in \mathbb{N}) .
$$

It can be easily verified from (1.8)

$$
\lambda(\omega)\left(M_{1}^{m}(a, b) \lambda(\omega)\right)^{\prime}=a M_{1}^{m}(a, b) \lambda(\omega)-(a+b) M_{1}^{m+1}(a, b) \lambda(\omega) \quad(b>0) .
$$

For more details see $[7-9,12,15,20,21,24]$. 
Definition 1.1. A function $\lambda(\omega)$ is subordinate to $\delta(\omega)$ in $U$ and written as: $\lambda(\omega) \prec \delta(\omega)$, if there exists a Schwarz function $k(\omega)$, which is holomorphic in $U^{*}$ with $|k(\omega)|<1$, such that $\lambda(\omega)=\delta(k(\omega))$. Furthermore, if the function $\delta(\omega)$ is univalent in $U^{*}$, then we have the following equivalence (see [22J):

$$
\lambda(\omega) \prec \delta(\omega) \text { and } \lambda(U) \subset \delta(U) .
$$

Further, $\lambda(\omega)$ is quasi-subordinate to $\delta(\omega)$ in $U^{*}$ and written as:

$$
\lambda(\omega) \prec_{q} \delta(\omega) \quad\left(\omega \in U^{*}\right),
$$

if there exist two analytic functions $\varphi(\omega)$ and $k(\omega)$ in $U^{*}$ such that $\frac{\lambda(\omega)}{\varphi(\omega)}$ is analytic in $U^{*}$ and

$$
|\varphi(\omega)| \leq 1 \text { and } k(\omega) \leq|\omega|<1 \quad \omega \in U^{*}
$$

satisfying

$$
\lambda(\omega)=\varphi(\omega) \delta(k(\omega)) \quad \omega \in U^{*} .
$$

Definition 1.2. For $-1 \leq S<T \leq 1$ the function $\lambda \in \sum_{p}$ is in the class $N_{p}^{m}(a, b ; d, S, T)$ if it satisfies the inequality

$$
1-\frac{1}{d}\left[\frac{\omega\left(M_{p}^{m}(a, b) \lambda(\omega)\right)^{\prime}}{M_{p}^{m}(a, b) \lambda(\omega)}+1\right] \prec \frac{1+S(\omega)}{1+T(\omega)},
$$

or, equivalently to:

$$
\left|\frac{\frac{\omega\left(M_{p}^{m}(a, b) \lambda(\omega)\right)^{\prime}}{M_{p}^{m}(a, b) \lambda(\omega)}+1}{T \frac{\omega\left(M_{p}^{m}(a, b) \lambda(\omega)\right)^{\prime}}{M_{p}^{m}(a, b) \lambda(\omega)}+[|d|(S-T)+T]}\right|<1 .
$$

Let $\sum_{p}^{*}$ denote the subclass of functions $\sum_{p}$ consisting of functions of the form:

$$
\lambda(\omega)=\frac{1}{\omega^{p}}+\sum_{t=p}^{\infty}\left|a_{t}\right| \omega^{t} \quad(p \in \mathbb{N}=\{1,2, \ldots\}) .
$$

Now, we define the class $N_{p}^{* m}(a, b ; d, S, T)=N_{p}^{m}(a, b ; d, S, T) \cap \sum_{p}^{*}$. For recent work on meromorphic functions we refer [10,11,13,14,17,19]. Motivated, from the above cited work we obtained the following results.

\section{Main Results}

In this section, in present the work to acquire sufficient conditions in which (1.13) gives the function $\lambda(\omega)$ within the class $N_{p}^{* m}(a, b ; d, S, T)$, as well as demonstrates that this condition is required for function which belong to this class. In our first theorem, we begin with the necessary and sufficient condition for function $\lambda$ in $N_{p}^{* m}(a, b ; d, S, T)$. We also prove some other related theorems. 
Theorem 2.1. Let the function $\lambda(\omega)$ is of the form (1.1). Then $\lambda(\omega) \in N_{p}^{* m}(a, b ; d, S, T)$ if and only if

$$
\sum_{t=p}^{\infty}\left[\frac{a}{a+b(p+t)}\right]^{m}\left|a_{t}\right|[1+t-\{|d|(S-T)+(1+t) T\}] \leq(1-p)(T-1)+|d|(S-T) .
$$

Proof. Assuming that (2.1) holds true, we obtain

$$
\begin{aligned}
& \left|\frac{\frac{\omega\left(M_{p}^{m}(a, b) \lambda(\omega)\right)^{\prime}}{M_{p}^{m}(a, b) \lambda(\omega)}+1}{T \frac{\omega\left(M_{p}^{m}(a, b) \lambda(\omega)\right)^{\prime}}{M_{p}^{m}(a, b) \lambda(\omega)}+[|d|(S-T)+T]}\right| \\
= & \left|\frac{\omega\left(M_{p}^{m}(a, b) \lambda(\omega)\right)^{\prime}+M_{p}^{m}(a, b) \lambda(\omega)}{T \omega\left(M_{p}^{m}(a, b) \lambda(\omega)\right)^{\prime}+[|d|(S-T)+T] M_{p}^{m}(a, b) \lambda(\omega)}\right| \\
= & \left|\frac{(1-p) \frac{1}{\omega^{p}}+\sum_{t=p}^{\infty}\left[\frac{a}{a+b(p+t)}\right]^{m} a_{t} \omega^{t}}{[(1-p) T+|d|(S-T)] \omega^{p}+\sum_{t=1}^{\infty}((t+1) T+|d|(S-T))\left|a_{t}\right| \omega^{t}}\right|<1 .
\end{aligned}
$$

Then, by maximum modulus theorem, we have $\lambda(\omega) \in N_{p}^{* m}(a, b ; d, S, T)$.

Conversely, assume that $\lambda(\omega)$ is in the class $N_{p}^{* m}(a, b ; d, S, T)$ with $\lambda(\omega)$ of the form (1.13), then we find from (1.12) that

$$
\begin{aligned}
& \left|\frac{\omega\left(M_{p}^{m}(a, b) \lambda(\omega)\right)^{\prime}+M_{p}^{m}(a, b) \lambda(\omega)}{T \omega\left(M_{p}^{m}(a, b) \lambda(\omega)\right)^{\prime}+[|d|(S-T)+T] M_{p}^{m}(a, b) \lambda(\omega)}\right|= \\
& \left|\frac{(1-p) \frac{1}{\omega^{p}}+\sum_{t=p}^{\infty}\left[\frac{a}{a+b(p+t)}\right]^{m} a_{t} \omega^{t}}{[(1-p) T+|d|(S-T)] \omega^{p}+\sum_{t=1}^{\infty}((t+1) T+|d|(S-T))\left|a_{t}\right| \omega^{t}}\right|<1,
\end{aligned}
$$

since the above inequality is genuine for all $\omega \in U$, let the value of $\omega$ on the real axis. Letting $\omega \longrightarrow 1^{-}$ through real values, we get

$$
\sum_{t=p}^{\infty}\left[\frac{a}{a+b(p+t)}\right]^{m}\left|a_{t}\right|[1+t-\{|d|(S-T)+(1+t) T\}] \leq(1-p)(T-1)+|d|(S-T) .
$$

Which complete the proof.

Corollary 2.1. If the function $\lambda(\omega)$ is of the form (1.1) is in the class $N_{p}^{* m}(a, b ; d, S, T)$ then

$$
\left|a_{t}\right| \leq \frac{(1-p)(T-1)+|d|(S-T)}{\sum_{t=p}^{\infty}\left[\frac{a}{a+b(p+t)}\right]^{m}[1+t-\{|d|(S-T)+(1+t) T\}]}, \quad(t \geq 1) .
$$

The result is sharp for the function

$$
\lambda(\omega)=\frac{1}{\omega^{p}}+\left(\frac{(1-p)(T-1)+|d|(S-T)}{\sum_{t=p}^{\infty}\left[\frac{a}{a+b(p+t)}\right]^{m}[1+t-\{|d|(S-T)+(1+t) T\}]}\right) \omega^{t} .
$$

Growth and distortion bounds for functions belonging to the class $N_{p}^{* m}(a, b ; d, S, T)$ will be given in the following result: 
Theorem 2.2. If a function $\lambda(\omega)$ given by (1.1) is in the class $N_{p}^{* m}(a, b ; d, S, T)$ then for $|\omega|=r$, we have:

$$
\begin{aligned}
& \frac{1}{r^{p}}-\left(\frac{(1-p)(T-1)+|d|(S-T)}{\left[\frac{a}{a+2 b p}\right]^{m}[2-\{|d|(S-T)+2 T\}]}\right) r \leq|\lambda(\omega)| \\
\leq & \frac{1}{r^{p}}+\left(\frac{(1-p)(T-1)+|d|(S-T)}{\left[\frac{a}{a+2 b p}\right]^{m}[2-\{|d|(S-T)+2 T\}]}\right) r,
\end{aligned}
$$

and

$$
\begin{array}{r}
\frac{-p}{|r|^{P+1}}-\left(\frac{(1-p)(T-1)+|d|(S-T)}{\left[\frac{a}{a+2 b p}\right]^{m}[2-\{|d|(S-T)+2 T\}]}\right) \\
\leq\left|\lambda^{\prime}(\omega)\right| \leq \frac{-p}{|r|^{p+1}}+\left(\frac{(1-p)(T-1)+|d|(S-T)}{\left[\frac{a}{a+2 b p}\right]^{m}[2-\{|d|(S-T)+2 T\}]}\right)
\end{array}
$$

Proof. In view of Theorem 2.2, we have

$$
\begin{aligned}
& {\left[\frac{a}{a+2 b p}\right]^{m}[2-\{|d|(S-T)+2 T\}] \sum_{t=p}^{\infty}\left|a_{t}\right| } \\
\leq & \sum_{t=p}^{\infty}\left[\frac{a}{a+b(p+t)}\right]^{m}\left|a_{t}\right|[1+t-\{|d|(S-T)+(1+t) T\}] \\
\leq & (1-p)(T-1)+|d|(S-T),
\end{aligned}
$$

which yield

$$
\sum_{t=p}^{\infty}\left|a_{t}\right| \leq \frac{(1-p)(T-1)+|d|(S-T)}{\left[\frac{a}{a+2 b p}\right]^{m}[2-\{|d|(S-T)+2 T\}]} \quad(t \in N)
$$

Therefore,

$$
|\lambda(\omega)| \leq \frac{1}{|\omega|^{p}}+|\omega| \sum_{t=p}^{\infty}\left|a_{t}\right| \leq \frac{1}{|\omega|^{p}}+|\omega| \frac{(1-p)(T-1)+|d|(S-T)}{\left[\frac{a}{a+2 b p}\right]^{m}[2-\{|d|(S-T)+2 T\}]}
$$

and

$$
|\lambda(\omega)| \geq \frac{1}{|\omega|^{p}}-|\omega| \sum_{t=p}^{\infty}\left|a_{t}\right| \leq \frac{1}{|\omega|^{p}}-|\omega| \frac{(1-p)(T-1)+|d|(S-T)}{\left[\frac{a}{a+2 b p}\right]^{m}[2-\{|d|(S-T)+2 T\}]} .
$$

Now, by differentiating(1.13), we have

$$
\left|\lambda^{\prime}(\omega)\right| \leq \frac{-p}{|\omega|^{p+1}}+\sum_{t=p}^{\infty}\left|a_{t}\right| \leq \frac{-p}{|\omega|^{p+1}}+\frac{(1-p)(T-1)+|d|(S-T)}{\left[\frac{a}{a+2 b p}\right]^{m}[2-\{|d|(S-T)+2 T\}]},
$$

and

$$
\left|\lambda^{\prime}(\omega)\right| \geq \frac{-p}{|\omega|^{p+1}}-\sum_{t=p}^{\infty}\left|a_{t}\right| \geq \frac{-p}{|\omega|^{p+1}}-\frac{(1-p)(T-1)+|d|(S-T)}{\left[\frac{a}{a+2 b p}\right]^{m}[2-\{|d|(S-T)+2 T\}]} .
$$


We have thus completed the proof.

Theorem 2.3. Let the function $\lambda(\omega)$ given by (1.13) is in the class $N_{p}^{* m}(a, b ; d, S, T)$. Then we have

(i) $\lambda$ is meromorphically starlike of order $q$ in the disc $|\omega|<r_{3}$, that is

$$
\Re\left(-\frac{\omega \lambda^{\prime}(\omega)}{\lambda(\omega)}\right)>q \quad\left(|\omega|<r_{3}, 0 \leq q<1\right),
$$

where

$$
r_{3}=\inf _{t \geq 1}\left[-\frac{\sum_{t=p}^{\infty}\left[\frac{a}{a+b(p+t)}\right]^{m}[1+t-\{|d|(S-T)+(1+t) T\}]}{(1-p)(T-1)+|d|(S-T)}\right]^{\frac{1}{t+p}} .
$$

(ii) $\lambda$ is meromorphically convex of order $q$ in the disc $|\omega|<r_{4}$, that is

$$
\Re\left\{-\left(1+\frac{\omega \lambda^{\prime \prime}(\omega)}{\lambda^{\prime}(\omega)}\right)\right\}>q \quad\left(|\omega|<r_{4}, 0 \leq q<1\right),
$$

where

$$
r_{4}=\inf _{t \geq 1}\left[\frac{\sum_{t=p}^{\infty}\left[\frac{a}{a+b(p+t)}\right]^{m}[1+t-\{|d|(S-T)+(1+t) T\}] p(1-q)}{(1-p)(T-1)+|d|(S-T)[t(1+q)]}\right]^{\frac{1}{t+p}}
$$

Proof. (i) In order to the inequality (2.9), we set

$$
\left|\frac{\frac{\omega \lambda^{\prime}(\omega)}{\lambda(\omega)}+1}{\frac{\omega \lambda^{\prime}(\omega)}{\lambda(\omega)}-1+2 q}\right| \leq \frac{(1-p)+\sum_{t=p}^{\infty}(t+1)\left|a_{t}\right||\omega|^{t+p}}{(2 q-p-1)+\sum_{t=1}^{\infty}(2 q-1+t)\left|a_{t}\right||\omega|^{t+p}} .
$$

Then we have

$$
\left|\frac{\frac{\omega \lambda^{\prime}(\omega)}{\lambda(\omega)}+1}{\frac{\omega \lambda^{\prime}(\omega)}{\lambda(\omega)}-1+2 q}\right| \leq 1 \quad(0 \leq q<1)
$$

if

$$
\sum_{t=1}^{\infty}\left|a_{t}\right||\omega|^{t+p} \leq-1
$$

Thus, by Theorem 2.1, the inequality (2.11) will be true if

$$
|\omega|^{t+p} \leq-\frac{\sum_{t=p}^{\infty}\left[\frac{a}{a+b(p+t)}\right]^{m}[1+t-\{|d|(S-T)+(1+t) T\}]}{(1-p)(T-1)+|d|(S-T)},
$$

then

$$
|\omega|=\left[-\frac{\sum_{t=p}^{\infty}\left[\frac{a}{a+b(p+t)}\right]^{m}[1+t-\{|d|(S-T)+(1+t) T\}]}{(1-p)(T-1)+|d|(S-T)}\right]^{\frac{1}{t+p}} .
$$

The last inequality leads us immediately to the disc $|\omega|<r_{3}$, where $r_{3}$ is given by (2.9). 
(ii) in order to prove the second affirmation of Theorem 2.3, we find from (1.1) that:

$$
\left|\frac{\frac{\omega \lambda^{\prime \prime}(\omega)}{\lambda^{\prime}(\omega)}+2}{\frac{\omega \lambda^{\prime \prime}(\omega)}{\lambda^{\prime}(\omega)}+2 q}\right| \leq \frac{p(p-1)+\sum_{t=p}^{\infty} t(t+1)\left|a_{t}\right||\omega|^{t+p}}{p(p+1-2 q)|\omega|^{p-1}+\sum_{t=p}^{\infty} t(t-1-2 q)\left|a_{t}\right||\omega|^{t+p}} .
$$

Thus we have desired inequality:

$$
\left|\frac{\frac{\omega \lambda^{\prime \prime}(\omega)}{\lambda^{\prime}(\omega)}+2}{\frac{\omega \lambda^{\prime \prime}(\omega)}{\lambda^{\prime}(\omega)}+2 q}\right| \leq 1 \quad(0 \leq q<1)
$$

if

$$
\sum_{t=1}^{\infty}\left(\frac{t(1+q)}{p(1-q)}\right)\left|a_{t}\right||\omega|^{t+1} \leq 1
$$

Thus, by Theorem 2.1, the inequality (2.12) will be true if

$$
\left(\frac{t(1+q)}{p(1-q)}\right)|\omega|^{t+p} \leq \frac{\sum_{t=p}^{\infty}\left[\frac{a}{a+b(p+t)}\right]^{m}[1+t-\{|d|(S-T)+(1+t) T\}]}{(1-p)(T-1)+|d|(S-T)}
$$

then

$$
|\omega|=\left[\frac{\sum_{t=p}^{\infty}\left[\frac{a}{a+b(p+t)}\right]^{m}[1+t-\{|d|(S-T)+(1+t) T\}] p(1-q)}{(1-p)(T-1)+|d|(S-T)[t(1+q)]}\right]^{\frac{1}{t+p}} .
$$

The last inequality readily yields the disc $|\omega|<r_{4}$, where $r_{4}$ is given by (2.10), which complete the proof.

Theorem 2.4. The class $N_{p}^{* m}(a, b ; d, S, T)$, is closed under convex linear combinations.

Proof. Let the function

$$
\lambda_{i}(\omega)=\frac{1}{\omega^{p}}+\sum_{t=p}^{\infty}\left|a_{t, i}\right| \omega^{t} \quad(i=1,2),
$$

are in $N_{p}^{* m}(a, b ; d, S, T)$, it suffices to show that the function $h$ defined by

$$
h(\omega)=(1-c) \lambda_{1}(\omega)+c \lambda_{2}(\omega) \quad(0 \leq c \leq 1)
$$

is in the class $N_{p}^{* m}(a, b ; d, S, T)$. Since

$$
h(\omega)=\frac{1}{\omega^{p}}+\sum_{t=p}^{\infty}\left[(1-c)\left|a_{t, 1}\right|+c\left|a_{t, 2}\right|\right] \omega^{t} \quad(0 \leq c \leq 1) .
$$


In view of Theorem 2.1, we have

$$
\begin{aligned}
& \sum_{t=p}^{\infty}\left[\frac{a}{a+b(p+t)}\right]^{m}[1+t-\{|d|(S-T)+(1+t) T\}]\left[(1-c)\left|a_{t, 1}\right|+c\left|a_{t, 2}\right|\right] \\
= & \sum_{t=p}^{\infty}\left[\frac{a}{a+b(p+t)}\right]^{m}[1+t-\{|d|(S-T)+(1+t) T\}](1-c)\left|a_{t, 1}\right| \\
& +\sum_{t=p}^{\infty}\left[\frac{a}{a+b(p+t)}\right]^{m}[1+t-\{|d|(S-T)+(1+t) T\}] c\left|a_{t, 2}\right| \\
\leq & (1-c)[(1-p)(T-1)+|d|(S-T)]+c[(1-p)(T-1)+|d|(S-T)] \\
= & {[(1-p)(T-1)+|d|(S-T)], }
\end{aligned}
$$

which show that $h(\omega) \in N_{p}^{* m}(a, b ; d, S, T)$, which is required.

Theorem 2.5. Let $\lambda_{0}(\omega)=\frac{1}{\omega^{p}}$ and

$$
\lambda_{t}(\omega)=\frac{1}{\omega}+\left(\frac{(1-p)(T-1)+|d|(S-T)}{\sum_{t=p}^{\infty}\left[\frac{a}{a+b(p+t)}\right]^{m}[1+t-\{|d|(S-T)+(1+t) T\}]}\right) \omega^{t} \quad t \geq 1
$$

then $\lambda \in N_{p}^{* m}(a, b ; d, S, T)$. If and only if it can be expressed in the form

$$
\lambda(\omega)=\sum_{t=p}^{\infty} v_{t} \lambda_{t}(\omega)
$$

where $v_{t} \geq 0$, and $\sum_{t=p}^{\infty} v_{t}=1$.

Proof. Let the function $\lambda(\omega)$ be expressed in the form given by $(2.13)$, then

$$
\lambda(\omega)=\frac{1}{\omega}+\left(v_{t} \frac{(1-p)(T-1)+|d|(S-T)}{\sum_{t=p}^{\infty}\left[\frac{a}{a+b(p+t)}\right]^{m}[1+t-\{|d|(S-T)+(1+t) T\}]}\right) \omega^{t}
$$

and for this function, we have

$$
\begin{aligned}
& \sum_{t=p}^{\infty}\left[\frac{a}{a+b(p+t)}\right]^{m}[1+t-\{|d|(S-T)+(1+t) T\}] \\
& \times v_{t} \frac{(1-p)(T-1)+|d|(S-T)}{\sum_{t=p}^{\infty}\left[\frac{a}{a+b(p+t)}\right]^{m}[1+t-\{|d|(S-T)+(1+t) T\}]} \omega^{t} \\
= & \sum_{t=p}^{\infty} v_{t}(1-p)(T-1)+|d|(S-T) \\
= & {\left[1-v_{0}\right](1-p)(T-1)+|d|(S-T) \leq(1-p)(T-1)+|d|(S-T), }
\end{aligned}
$$


the condition (2.1) is satisfied. Thus, $\lambda \in N_{p}^{* m}(a, b ; d, S, T)$. Conversely, we suppose that $\lambda \in$ $N_{p}^{* m}(a, b ; d, S, T)$. Since

$$
\left|a_{t}\right| \leq \frac{(1-p)(T-1)+|d|(S-T)}{\sum_{t=p}^{\infty}\left[\frac{a}{a+b(p+t)}\right]^{m}[1+t-\{|d|(S-T)+(1+t) T\}]}, \quad(t \geq 1)
$$

we set

$$
v_{t}=\frac{\sum_{t=p}^{\infty}\left[\frac{a}{a+b(p+t)}\right]^{m}[1+t-\{|d|(S-T)+(1+t) T\}]}{(1-p)(T-1)+|d|(S-T)}\left|a_{t}\right| \quad(t \geq 1),
$$

and

$$
v_{0}=1-\sum_{t=p}^{\infty} v_{t}
$$

so it follows that

$$
\lambda(\omega)=\sum_{t=p}^{\infty} v_{t} \lambda_{t}(\omega) .
$$

This completes the assertion of Theorem 2.5.

\section{Conclusion}

In our current investigation, we have presented and studied thoroughly some new subclasses of $p-$ valent functions related with meromorphic convex and meromorphic starlike functions, in connection with the integral operator given by (1.7). We have obtained sufficient and necessary conditions in relation to these classes, including growth and distortion theorem along with a radius problem. The technique and ideas of this paper may stimulate further research in the theory of multivalent meromorphic functions.

Conflicts of Interest: The author(s) declare that there are no conflicts of interest regarding the publication of this paper.

\section{REFERENCES}

[1] M. K. Aouf and T. M. Seoudy, Some properties of a certain subclass of multivalent analytic functions involving the Liu-Owa operator. Comput. Math. Appl. 60 (2010), 1525-1535.

[2] M. K. Aouf and T. M. Seoudy, Some preserving subordination and super ordination of analytic functions involving the Liu-Owa integral operator, Comput. Math. Appl. 62 (2011), 3575-3580.

[3] M. K. Aouf and T. M. Seoudy, Some preserving subordination and superordination of the Liu-Owa integral operator, Complex Anal. Oper. Theory. 7 (2013), 275-283.

[4] K. R. Alhindi and M. Darus, A New Class of Meromorphic Functions Involving the Polylogarithm Function, J. Complex Anal. 2014 (2014), 864805.

[5] R. Bharati, R. Parvatham and A. Swaminathan, On subclasses of uniformly convex functions and corresponding class of starlike functions, Tamkang J. Math. 28 (1997), 17-32.

[6] N. E. Cho and S. Owa, Sufficient conditions for meromorphic starlikeness and close to-convexity of order $\alpha$, Int. J. Math. Sci. 26 (2001), 317-319. 
[7] M. Darus, S. Hussain, M. Raza and J. Sokol, On a subclass of starlike functions, Res. Math. 73 (2018), 22.

[8] H. Aldweby and M. Darus, On Harmonic Meromorphic Functions Associated with Basic Hypergeometric Functions, Sci. World J. 2013 (2013), 164287.

[9] R. M. El-Ashwah, M. K. Aouf, A. A. Hassan and A. H. Hassan, Certain new classes of analytic functions with varying arguments, J. Complex Anal. 2013 (2013), Art. ID 958210.

[10] E. A. Elrifai, H. E. Darwish and A. R. Ahmed, On certain subclasses of meromorphic functions associated with certain differential operators. Appl. Math. Lett. 25 (2012), 952-958.

[11] S. Elhaddad and M. Darus, On meromorphic functions defined by a new operator containing the Mittag-Leffler fuction, Symmetry. 11 (2019), Art. ID 210.

[12] B. A. Frasin, M. Darus, On Certain Meromorphic Functions with Positive Coefficients, Southeast Asian Bull. Math. 28 (2004), 615-623.

[13] F. Ghanim, M. Darus, On Class of Hypergeometric Meromorphic Functions with Fixed Second Positive Coefficients, Gen. Math.17 (2009), 13-28.

[14] H. M. Hossen, H. M. Srivastava and M. K. Aouf, A unified presentation of some classes of meromorphically multivalent functions. Comput. Math. Appl. 38 (1999), 63-70.

[15] W. Janowski, Some extrenal problems for certain families of analytic functions, Ann. Pol. Math. 28 (1973), $297-326$.

[16] W. Kaplan, Close-to-convex schlicht functions, Michigan Math. J. 1 (1952), 169-185.

[17] A. Y. Lashin, On certain subclasses of meromorphic functions associated with certain integral operators. Comput. Math. Appl. 59 (2010), 524-531.

[18] J. L. Liu and H. M. Srivastava, Classes of meromorphically multivalent functions associated with the generalized hyper geometric function. Math. Comput. Model. 39 (2004), 21-34.

[19] J. L. Liu and S. Owa, Properties of certain integral operators, Int. J.Math. Math. Sci. 3 (2004), 69-75.

[20] J. E. Miller, Convex meromrphic mapping and related functions, Proc. Amer. Math. Soc. 25 (1970), 220-228.

[21] A. Mannino, Some inequalities concerning starlike and convex functions, Gen. Math. 12 (2004), 5-12.

[22] S. S. Miller and P. T. Mocanu, Differential Subordinations Theory and Applications, in Monographs and Textbooks in Pure and Applied Mathematics, 225, Marcel Dekker, New York, 2000.

[23] M. S. Roberston, On the theory of univalent functions. Annal. Math. 37 (1936), 374-408.

[24] A. Rasheed, S. Hussain, S. G. A. Shah, M. Darus and S. Lodhi, Majorization problem for two subclasses of meromorphic functions associated with a convolution operator, AIMS Math. 5(2020), 5157-5170. 